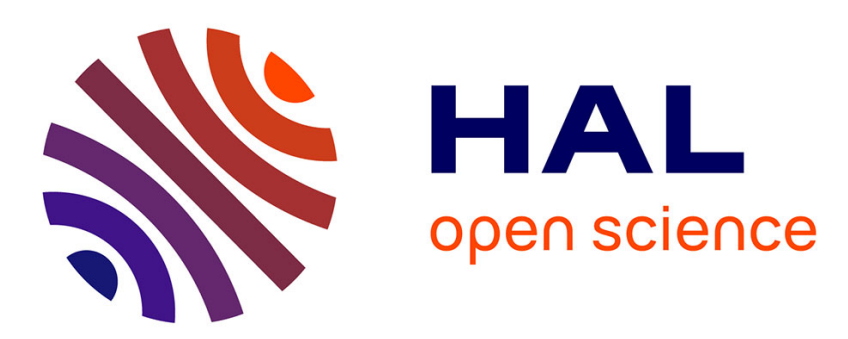

\title{
Numerical Simulations of the Inviscid Primitive Equations in a Limited Domain
}

\author{
Antoine Rousseau, Roger M. Temam, Joe Tribbia
}

\section{To cite this version:}

Antoine Rousseau, Roger M. Temam, Joe Tribbia. Numerical Simulations of the Inviscid Primitive Equations in a Limited Domain. Mathematical Methods for Hydrodynamics, Université Lille 1, Jun 2005, Lille, France. pp.163-181, 10.1007/978-3-7643-7742-7_10 . inria-00172561

\section{HAL Id: inria-00172561 \\ https://hal.inria.fr/inria-00172561}

Submitted on 17 Sep 2007

HAL is a multi-disciplinary open access archive for the deposit and dissemination of scientific research documents, whether they are published or not. The documents may come from teaching and research institutions in France or abroad, or from public or private research centers.
L'archive ouverte pluridisciplinaire HAL, est destinée au dépôt et à la diffusion de documents scientifiques de niveau recherche, publiés ou non, émanant des établissements d'enseignement et de recherche français ou étrangers, des laboratoires publics ou privés. 
Accepté pour publication dans

Proceedings of Mathematical Methods for Hydrodynamics

Juin 2005, University of Lille, France

\title{
NUMERICAL SIMULATIONS OF THE INVISCID PRIMITIVE EQUATIONS IN A LIMITED DOMAIN
}

\author{
A. ROUSSEAU ${ }^{b}$, R. TEMAM ${ }^{b *}$, AND J. TRIBBIA $^{\sharp}$ \\ 'Laboratoire d'Analyse Numérique, Université Paris-Sud, Orsay, France. \\ *The Institute for Scientific Computing and Applied Mathematics, \\ Indiana University, Bloomington, IN, USA. \\ \#National Center for Atmospheric Research, Boulder, Colorado, USA.
}

\begin{abstract}
This work is dedicated to the numerical computations of the primitive equations (PEs) of the ocean without viscosity with the nonlocal (mode by mode) boundary conditions introduced in [RTT05b]. We consider the 2D nonlinear PEs, and firstly compute the solutions in a "large" rectangular domain $\Omega_{0}$ with periodic boundary conditions in the horizontal direction. Then we consider a subdomain $\Omega_{1}$, in which we compute a second numerical solution with transparent boundary conditions. Two objectives are achieved. On the one hand the absence of blow-up in these computations indicates that the PEs without viscosity are well-posed when supplemented with the boundary conditions introduced in [RTT05b]. On the other hand they show a very good coincidence on the subdomain $\Omega_{1}$ of the two solutions, thus showing also the computational relevance of these new boundary conditions. We end this study with some numerical simulations of the linearized primitive equations, which correspond to the theoretical results established in [RTT05b], and evidence the transparent properties of the boundary conditions.
\end{abstract}

\section{Introduction : Motivations AND ObJECTIVES}

In this work, we intend to present our numerical simulations of the $2 \mathrm{D}$ primitive equations (PEs) without viscosity supplemented with the nonreflective and nonlocal boundary conditions introduced in [RTT05b]. To this aim, we first compute the PEs in a "large" $(x, z)$ domain $\Omega_{0}=(0, L) \times(-H, 0)$, with no-flux boundary conditions at top and bottom, and periodic boundary conditions in the horizontal direction 
(see Figure 1).

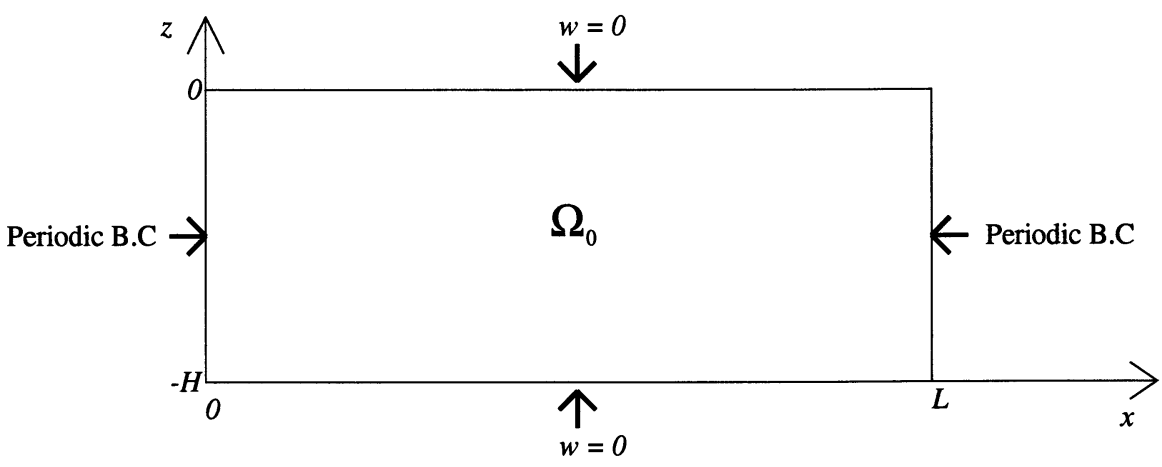

FiguRE 1. Domain $\Omega_{0}$

We then consider a sub-domain $\Omega_{1}=(a, b) \times(-H, 0)$, where $0<a<$ $b<L$, so that $\Omega_{1} \subset \Omega_{0}$. We perform numerical simulations of the PEs on $\Omega_{1}$, with the no-flux boundary condition at top and bottom, but we use the transparent boundary conditions at $x=a$ and $x=b$ (the actual values are taken taken from the calculations in $\left.\Omega_{0}\right)$. The initial condition is the same as on $\Omega_{0}$ (restriction to $\Omega_{1}$ ):

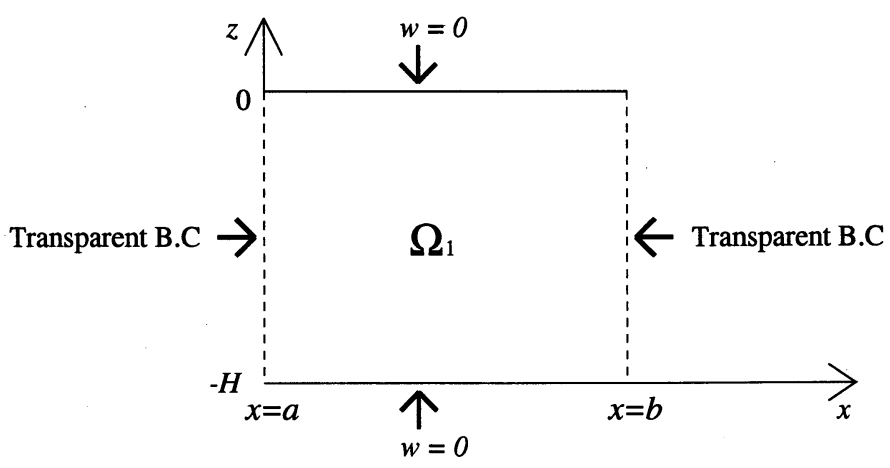

FiguRE 2. Subdomain $\Omega_{1}$

Our objectives are twofold: firstly in view of extending the theoretical results of [RTT05b] to the nonlinear PEs, we test boundary conditions similar to those in [RTT05b]; secondly to show that the proposed boundary conditions are well-suited for the problem of numerical simulations in a limited domain. This is done by observing that the solutions computed on $\Omega_{1}$ only (with the nonreflecting boundary conditions) match well, on $\Omega_{1}$, with the solutions computed on the whole domain $\Omega_{0}$. 
This article is organized as follows. In Section 2, we present the equations and the so-called normal mode expansion. Then we introduce in Section 3 the numerical schemes that we use in order to solve the primitive equations (2.1). The spectral method is employed for the vertical direction, whereas the $x$ and $t$ derivatives are discretized using finite differences. In Section 4, we present the numerical computations of the nonlinear PEs. The first results are dedicated to the periodic boundary conditions for $\Omega_{0}$; then we implement the nonreflective boundary conditions introduced in [RTT05b] for $\Omega_{1}$, and we end this section with a comparison between the two different numerical solutions.

\section{The Equations AND The Normal Expansion}

In this study we consider the nonlinear primitive equations without viscosity, independant of $y$, see e.g. [RTT05b]:

$$
\begin{aligned}
\frac{\partial u}{\partial t}+\left(\bar{U}_{0}+u\right) \frac{\partial u}{\partial x}+w \frac{\partial u}{\partial z}-f v+\frac{\partial \phi}{\partial x} & =F_{u} \\
\frac{\partial v}{\partial t}+\left(\bar{U}_{0}+u\right) \frac{\partial v}{\partial x}+w \frac{\partial v}{\partial z}+f u & =F_{v}-f \bar{U}_{0} \\
\frac{\partial \psi}{\partial t}+\left(\bar{U}_{0}+u\right) \frac{\partial \psi}{\partial x}+\left(N^{2}+\frac{\partial \psi}{\partial z}\right) w & =F_{\psi} \\
\frac{\partial \phi}{\partial z}=-\frac{\rho}{\rho_{0}} g & =\psi \\
\frac{\partial u}{\partial x}+\frac{\partial w}{\partial z} & =0
\end{aligned}
$$

We consider the equations in the bidimensional domain $\Omega_{0}=(0, L) \times$ $(-H, 0)$, and supplement them with an initial data $u_{0}, v_{0}, \psi_{0}$. The boundary condition taken at $z=-H$ and $z=0$ is classically $w=$ 0 , and we will consider in this study two different sets of boundary conditions in the horizontal direction: the periodic ones and the nonreflective ones.

2.1. The Linearized Primitive Equations. We first start with the linearized version of (2.1) on which the study of (2.1) is based. The 
linearization of the PEs (2.1) around the steady flow $\left(\bar{U}_{0}, 0,0\right)$ reads

$$
\begin{aligned}
\frac{\partial u}{\partial t}+\bar{U}_{0} \frac{\partial u}{\partial x}-f v+\frac{\partial \phi}{\partial x} & =F_{u} \\
\frac{\partial v}{\partial t}+\bar{U}_{0} \frac{\partial v}{\partial x}+f u & =F_{v}-f \bar{U}_{0} \\
\frac{\partial \psi}{\partial t}+\bar{U}_{0} \frac{\partial \psi}{\partial x}+N^{2} w & =F_{\psi} \\
\frac{\partial \phi}{\partial z}=-\frac{\rho}{\rho_{0}} g & =\psi \\
\frac{\partial u}{\partial x}+\frac{\partial w}{\partial z} & =0
\end{aligned}
$$

where $N$ is the so-called buoyancy frequency, assumed to be constant. In this study, we assume that the constant $\bar{U}_{0}$ is positive. Naturally, this hypothesis is not restrictive and the study could easily be extended to the case where $\bar{U}_{0}$ is negative.

Classically we proceed by separation of variables and actually look for the unknown functions $(u, v, w, \phi, \psi)$ under the form (see [RTT05b] for more details):

$$
(u, v, \phi)=\mathcal{U}(z)(\hat{u}, \hat{v}, \hat{\phi})(x, t), \quad(w, \psi)=\mathcal{W}(z)(\hat{w}, \hat{\psi})(x, t),
$$

where the functions $\hat{u}, \hat{v}, \hat{w}$, and $\hat{\phi}$ only depend on $x$ and $t$. Introducing the decomposition (2.3) into equations (2.2) shows that $\mathcal{W}$ (and then $\mathcal{U})$ solves a two-point eigenvalue problem with the boundary condition $\mathcal{W}=0$ at top and bottom $(z=0,-H)$. We thus obtain the normal modes $\mathcal{W}_{m}(z)$ and $\mathcal{U}_{m}(z)$ such that

$$
\begin{aligned}
\mathcal{U}_{m}(z) & =\sqrt{\frac{2}{H}} \cos \left(N \lambda_{m} z\right), \quad \mathcal{U}_{0}(z)=\frac{1}{\sqrt{H}} \\
\mathcal{W}_{m}(z) & =\sqrt{\frac{2}{H}} \sin \left(N \lambda_{m} z\right), \\
\lambda_{m} & =\frac{m \pi}{N H} .
\end{aligned}
$$

We then look for the general solution in the form a series

$$
\begin{aligned}
(u, v, \phi) & =\sum_{m \geq 0} \mathcal{U}_{m}(z)\left(\hat{u}_{m}, \hat{v}_{m}, \hat{\phi}_{m}\right)(x, t) \\
(w, \psi) & =\sum_{m \geq 1} \mathcal{W}_{m}(z)\left(\hat{w}_{m}, \hat{\psi}_{m}\right)(x, t)
\end{aligned}
$$


We notice that $\forall m^{\prime} \geq 0, m \geq 1$, we have the usual orthogonality properties:

$$
\left\{\begin{aligned}
\int_{-L_{3}}^{0} \mathcal{U}_{m^{\prime}}(z) \mathcal{U}_{m}(z) d z & =\delta_{m^{\prime}, m} \\
\int_{-L_{3}}^{0} \mathcal{U}_{m^{\prime}}(z) \mathcal{W}_{m}(z) d z & =0 \\
\mathcal{U}_{m}^{\prime}(z) & =-N \lambda_{m} \mathcal{W}_{m}(z) \\
\mathcal{W}_{m}^{\prime}(z) & =N \lambda_{m} \mathcal{U}_{m}(z)
\end{aligned}\right.
$$

In the numerical simulations, we will truncate the series after $M$ terms. Naturally, the larger $M$ is, the more accurate the method is expected to be, but the heavier the computations are. Typically, $M=10$ is satisfying from the physical point of view.

The case of the steady mode $m=0$ is very simple, and is explained in [RTT05b]. From now on we only consider the modes $m \geq 1$.

Writing the linear PEs mode by mode, and writing $\left(u_{m}, v_{m}, w_{m}, \psi_{m}\right.$, $\left.\phi_{m}\right)$ instead of $\left(\hat{u}_{m}, \hat{v}_{m}, \hat{w}_{m}, \hat{\psi}_{m}, \hat{\phi}_{m}\right)$, we obtain the following system of integrodifferential equations $(1 \leq m \leq M)$ :

$$
\begin{gathered}
\frac{\partial u_{m}}{\partial t}+\bar{U}_{0} \frac{\partial u_{m}}{\partial x}-f v_{m}+\frac{\partial \phi_{m}}{\partial x}=F_{u, m} \\
\frac{\partial v_{m}}{\partial t}+\bar{U}_{0} \frac{\partial v_{m}}{\partial x}+f u_{m}=F_{v, m} \\
\frac{\partial \psi_{m}}{\partial t}+\bar{U}_{0} \frac{\partial \psi_{m}}{\partial x}+N^{2} w_{m}=F_{\psi, m} \\
\phi_{m}=-\frac{\psi_{m}}{N \lambda_{m}} \\
w_{m}=-\frac{1}{N \lambda_{m}} \frac{\partial u_{m}}{\partial x}
\end{gathered}
$$

Taking equations (2.10d) and (2.10e) into account, equations (2.10a)(2.10c) become:

$$
\begin{array}{r}
\frac{\partial u_{m}}{\partial t}+\bar{U}_{0} \frac{\partial u_{m}}{\partial x}-f v_{m}-\frac{1}{N \lambda_{m}} \frac{\partial \psi_{m}}{\partial x}=F_{u, m} \\
\frac{\partial v_{m}}{\partial t}+\bar{U}_{0} \frac{\partial v_{m}}{\partial x}+f u_{m}=F_{v, m} \\
\frac{\partial \psi_{m}}{\partial t}+\bar{U}_{0} \frac{\partial \psi_{m}}{\partial x}-\frac{N}{\lambda_{m}} \frac{\partial u_{m}}{\partial x}=F_{\psi, m}
\end{array}
$$


We have $M$ systems of three coupled integro-differential equations (time dependant with one space variable). We will discretize this system in Section 3 below.

For every $m \leq M$, we introduce $\xi_{m}=u_{m}-\psi_{m} / N, \eta_{m}=u_{m}+\psi_{m} / N$, and let $v_{m}$ unchanged. In terms of these variables, the system (2.10) becomes:

$$
\begin{gathered}
\frac{\partial \xi_{m}}{\partial t}+\left(\bar{U}_{0}+\frac{1}{\lambda_{m}}\right) \frac{\partial \xi_{m}}{\partial x}-f v_{m}=F_{\xi, m} \\
\frac{\partial v_{m}}{\partial t}+\bar{U}_{0} \frac{\partial v_{m}}{\partial x}+f \frac{\xi_{m}+\eta_{m}}{2}=F_{v, m} \\
\frac{\partial \eta_{m}}{\partial t}+\left(\bar{U}_{0}-\frac{1}{\lambda_{m}}\right) \frac{\partial \eta_{m}}{\partial x}-f v_{m}=F_{\eta, m} .
\end{gathered}
$$

The physical quantities can be obtained from $\xi_{m}, \eta_{m}$ and $v_{m}$ with:

$$
\begin{array}{r}
u_{m}(x, t)=\frac{\xi_{m}+\eta_{m}}{2}(x, t), \\
w_{m}(x, t)=-\frac{u_{m_{x}}}{N \lambda_{m}}(x, t), \\
\psi_{m}(x, t)=\frac{N\left(\eta_{m}-\xi_{m}\right)}{2}(x, t), \\
\phi_{m}(x, t)=-\frac{\psi_{m}}{N \lambda_{m}}(x, t) .
\end{array}
$$

It is crucial to notice that $\bar{U}_{0}, \bar{U}_{0}+1 / \lambda_{m}$ are always positive, whereas $\bar{U}_{0}-1 / \lambda_{m}$ can either be positive or negative ${ }^{1}$, depending on the value of $m \leq M$; actually, the sign of these three characteristic values will determine the way we discretize the equations (2.12) in the horizontal direction.

Thanks to (2.6), there exists a critical value $m_{c}$ such that $\bar{U}_{0}-1 / \lambda_{m}$ is negative (resp. positive) if $m \leq m_{c}$ (resp. $m>m_{c}$ ). The corresponding modes are then called subcritical (resp. supercritical).

2.2. Boundary Conditions for the Linear Case. We now recall the boundary conditions first introduced in [RTT05b]. The subcritical modes $\left(m \leq m_{c}\right)$ and the supercritical ones $\left(m>m_{c}\right)$ have to be

\footnotetext{
${ }^{1}$ This is actually why the PEs in a bounded domain are ill-posed with any set of local boundary conditions, see [OS78, TT03, PR05, RTT05a, RTT05b].
} 
NUMERICAL SIMULATIONS OF THE INVISCID PRIMITIVE EQUATIONS 7 handled separately. Namely, we prescribe for $m \leq m_{c}$ :

$$
\begin{aligned}
\xi_{m}(0, t) & =\xi_{m, g}^{l}(t), \\
v_{m}(0, t) & =v_{m, g}^{l}(t), \\
\eta_{m}(L, t) & =\eta_{m, g}^{r}(t),
\end{aligned}
$$

where the quantities $\xi_{m, g}^{l}, v_{m, g}^{l}$ and $\eta_{m, g}^{r}$ are some given functions that depend on time. The boundary conditions for the supercritical modes $\left(m>m_{c}\right)$ are

$$
\begin{aligned}
& \xi_{m}(0, t)=\xi_{m, g}^{l}(t), \\
& v_{m}(0, t)=v_{m, g}^{l}(t), \\
& \eta_{m}(0, t)=\eta_{m, g}^{0}(t) .
\end{aligned}
$$

where $\xi_{m, g}^{l}, v_{m, g}^{l}$ and $\eta_{m, g}^{l}$ are also given. We notice that (2.14) differ from (2.15), since two characteristics (resp. three) enter the domain at $x=0$ (resp. $x=L$ ) for subcritical modes (resp. the supercritical ones).

From the continuous viewpoint, the boundary conditions (2.14) can be written in the form of integral equations:

$$
\begin{array}{r}
\int_{-L_{3}}^{0} u(0, z) \mathcal{U}_{m}(z) d z-\frac{1}{N} \int_{-L_{3}}^{0} \psi(0, z) \mathcal{W}_{m}(z) d z=0 \\
\int_{-L_{3}}^{0} v(0, z) \mathcal{U}_{m}(z) d z=0 \\
\int_{-L_{3}}^{0} u\left(L_{1}, z\right) \mathcal{U}_{m}(z) d z+\frac{1}{N} \int_{-L_{3}}^{0} \psi\left(L_{1}, z\right) \mathcal{W}_{m}(z) d z=0
\end{array}
$$

The supercritical boundary conditions (2.15) can similarly be rewritten in the form of an infinite sequence of integral equations:

$$
\begin{gathered}
\int_{-L_{3}}^{0} u(0, z) \mathcal{U}_{m}(z) d z=0 \\
\int_{-L_{3}}^{0} v(0, z) \mathcal{U}_{m}(z) d z=0 \\
\int_{-L_{3}}^{0} \psi(0, z) \mathcal{W}_{m}(z) d z=0
\end{gathered}
$$

We recall that the linearized PEs (2.2) supplemented with the boundary conditions (2.16)-(2.16) lead to a well-posed problem. See [RTT05b] for more details. 
2.3. The Modal Form of the Nonlinear Primitive Equations. We return to equations (2.1), and perform the same normal mode decomposition. We obtain the nonlinear form of equations (2.10a)(2.10c), (2.10d) and (2.10e) beeing unchanged. For $1 \leq m \leq M$ we have:

$$
\begin{array}{r}
\frac{\partial u_{m}}{\partial t}+\bar{U}_{0} \frac{\partial u_{m}}{\partial x}-f v_{m}+\frac{\partial \phi_{m}}{\partial x}+B_{u, m}(U)=F_{u, m} \\
\frac{\partial v_{m}}{\partial t}+\bar{U}_{0} \frac{\partial v_{m}}{\partial x}+f u_{m}+B_{v, m}(U)=F_{v, m} \\
\frac{\partial \psi_{m}}{\partial t}+\bar{U}_{0} \frac{\partial \psi_{m}}{\partial x}+N^{2} w_{m}+B_{\psi, m}(U)=F_{\psi, m},
\end{array}
$$

where $B_{u, m}, B_{v, m}$ and $B_{\psi, m}$ are the following modal parts of the nonlinearities:

$$
\begin{aligned}
B_{u, m} & =\int_{-H}^{0}\left(u \frac{\partial u}{\partial x}+w \frac{\partial u}{\partial z}\right) \mathcal{U}_{m} d z \\
B_{v, m} & =\int_{-H}^{0}\left(u \frac{\partial v}{\partial x}+w \frac{\partial v}{\partial z}\right) \mathcal{U}_{m} d z \\
B_{\psi, m} & =\int_{-H}^{0}\left(u \frac{\partial \psi}{\partial x}+w \frac{\partial \psi}{\partial z}\right) \mathcal{W}_{m} d z
\end{aligned}
$$

with $u, v, \psi, w$ truncated to $M$ modes.

2.4. Boundary Conditions for the Nonlinear Case. We make the same change of variables $\xi_{m}=u_{m}-\psi_{m} / N, \eta_{m}=u_{m}+\psi_{m} / N$, and obtain the nonlinear version of (2.12), namely:

$$
\begin{gathered}
\frac{\partial \xi_{m}}{\partial t}+\left(\bar{U}_{0}+\frac{1}{\lambda_{m}}\right) \frac{\partial \xi_{m}}{\partial x}-f v_{m}+B_{\xi, m}(U)=F_{\xi, m} \\
\frac{\partial v_{m}}{\partial t}+\bar{U}_{0} \frac{\partial v_{m}}{\partial x}+f \frac{\xi_{m}+\eta_{m}}{2}+B_{v, m}(U)=F_{v, m} \\
\frac{\partial \eta_{m}}{\partial t}+\left(\bar{U}_{0}-\frac{1}{\lambda_{m}}\right) \frac{\partial \eta_{m}}{\partial x}-f v_{m}+B_{\eta, m}(U)=F_{\eta, m}
\end{gathered}
$$

where $B_{\xi, m}=B_{u, m}-B_{\psi, m} / N$ and $B_{\eta, m}=B_{u, m}+B_{\psi, m} / N$.

We assume in the following that the initial data is such that the nonlinear part is small compared to the stratified flow $\left(\bar{U}_{0}, 0,0\right)$, so that the characteristic values do not change sign, at least during a certain period of time. Assuming so, we conjecture that the boundary conditions provided for the linearized system will give a well-posed problem for 
the nonlinear equations, at least for some time. We leave the theoretical analysis to subsequent studies, and perform here the corresponding numerical simulations based on this hypothesis, which is conforted by the lack of numerical blow-up. Hence the boundary conditions that we consider for the nonlinear case are (2.16)-(2.17).

\section{Numerical SCHEME}

3.1. Vertical Discretization by Spectral Method. In the vertical direction, we proceed by normal modes decomposition as in (2.7), (2.8). From the numerical point of view, we will need to transform some griddata into modal coefficients in the $\mathcal{U}_{m}$ or $\mathcal{W}_{m}$ bases of $L^{2}(-H, 0)$, and vice versa.

Given a function $f$ represented by its values $f_{l}$ on a grid $z_{l}=-H+l \Delta z$, $0 \leq l \leq l_{\max }, \Delta z=H / l_{\text {max }}$, we want to transform it into coefficients $f_{m}, 0 \leq m \leq M$. To this aim we use the second order central point integration method, with the $z_{l}$ as collocation points. For the functions $u, v$ and $\phi$, we decompose them in the $\mathcal{U}_{m}$ basis of $L^{2}(-H, 0)$. For $0 \leq m \leq M$ :

$$
\left\{u_{m}, v_{m}, \phi_{m}\right\}=\Delta z \sum_{l=0}^{l_{\max }-1} \frac{\mathcal{U}_{m}\left(z_{l}\right) \cdot\{u, v, \phi\}\left(z_{l}\right)+\mathcal{U}_{m}\left(z_{l+1}\right) \cdot\{u, v, \phi\}\left(z_{l+1}\right)}{2}
$$

and for $w$ and $\psi, 1 \leq m \leq M$ :

$$
\left\{w_{m}, \psi_{m}\right\}=\Delta z \sum_{l=0}^{l_{\max }-1} \frac{\mathcal{W}_{m}\left(z_{l}\right) \cdot\{w, \psi\}\left(z_{l}\right)+\mathcal{W}_{m}\left(z_{l+1}\right) \cdot\{w, \psi\}\left(z_{l+1}\right)}{2}
$$

This approach which is that proposed by the physicists is different from the more mathematical approach to spectral and pseudo-spectral methods (as in e.g. [BM97, GH01]). The advantage of such a choice is that the orthogonality relations (2.9) are satisfied from the numerical point of view. Further studies and comparisons of the two approaches will be needed in the future.

On the contrary, if the function is given by its modal coefficients, the values on the $z$-grid $z_{l}, 0 \leq l \leq l_{\max }$ is simply given by: 


$$
\begin{array}{r}
(u, v, \phi)\left(z_{l}\right)=\sum_{m=0}^{M}\left(u_{m}, v_{m}, \phi_{m}\right) \mathcal{U}_{m}\left(z_{l}\right) \\
(w, \psi)\left(z_{l}\right)=\sum_{m=0}^{M}\left(w_{m}, \psi_{m}\right) \mathcal{W}_{m}\left(z_{l}\right)
\end{array}
$$

In the numerical simulations, we are given some initial data on the physical grid $\left(z_{l}\right)_{0 \leq l \leq l_{\max }}$. We transform them into modal coefficients thanks to formulas (3.1) or (3.2), and if the problem is linear, we keep them all along the computations, except for graphic purposes, for which we use inverse formulas (3.3)-(3.4) to return to physical space. Naturally, in the nonlinear case, we need to operate (3.1)-(3.4) once at every time step, in order to avoid the computation of a convolution product, that would cost too much in term of CPU time and is not considered an appropriate numerical procedure. We compute the nonlinear terms of the equations in the physical space $(x, z)$ thanks to Fourier and inverse Fourier transforms.

\subsection{Finite Differences in Time and Space (Horizontal Direc-} tion). Looking at the form of (2.20), we choose to discretize these equations in the horizontal direction with the finite differences method. Naturally, care has to be taken to the sign of the characteristic values, in order to take an upwind (hence stable) spatial discretization of the $x$-derivative. Whereas $\bar{U}_{0}$ and $\bar{U}_{0}+1 / \lambda_{m}$ are always positive, the third characteristic value of the $m t h$ mode - in the linear case - is $\bar{U}_{0}-1 / \lambda_{m}$. and can either be positive or negative for the actual physical values that we consider.

In the non linear case, since the initial data is small compared to $\bar{U}_{0}$ ([TT03]), we implicitly assume that $m_{c}$ remains unchanged for a certain period of time. Until a full nonlinear theory is performed, a first step in the verification of this hypothesis would be to linearize Equation (2.18) (or (2.11)-(2.20)) around the current state which may amount to replacing $\bar{U}_{0}$ by $\bar{U}_{0}+u$, but may also involve a more complex analysis already in the linearized case. These involved issues are investigated in a work in progress. 
That is, for every subcritical mode $m \leq m_{c}$, we discretize (2.20) as follows:

$$
\frac{\xi_{m, j}^{n+1}-\xi_{m, j}^{n}}{\Delta t^{n}}+\left(\bar{U}_{0}+\frac{1}{\lambda_{m}}\right) \frac{\xi_{m, j}^{n}-\xi_{m, j-1}^{n}}{\Delta x}-f v_{m, j}^{n}=F_{\xi, m, j}^{n}-B_{\xi, m, j}^{n},
$$

$$
\frac{v_{m, j}^{n+1}-v_{m, j}^{n}}{\Delta t^{n}}+\bar{U}_{0} \frac{v_{m, j}^{n}-v_{m, j-1}^{n}}{\Delta x}+f \frac{\xi_{m, j}^{n}+\eta_{m, j}^{n}}{2}=F_{v, m, j}^{n}-B_{v, m, j}^{n}
$$

$$
\frac{\eta_{m, j}^{n+1}-\eta_{m, j}^{n}}{\Delta t^{n}}+\left(\bar{U}_{0}-\frac{1}{\lambda_{m}}\right) \frac{\eta_{m, j+1}^{n}-\eta_{m, j}^{n}}{\Delta x}-f v_{m, j}^{n}=F_{\eta, m, j}^{n}-B_{\eta, m, j}^{n}
$$

where the right-hand-side of (3.5) contains the nonlinear terms, computed explicitly thanks to an Adams-Bashforth scheme.

Equations (3.5a) and(3.5b) hold for $1 \leq j \leq J$, whereas (3.5c) is written for $0 \leq j \leq J-1$. There are no equations for $\xi_{m, 0}^{n+1}, v_{m, 0}^{n+1}$ and $\eta_{m, J}^{n+1}$, these quantities being given by the boundary conditions as required in [RTT05b]. On the contrary, if $m>m_{c}$ (supercritical case), we choose for $1 \leq j \leq J$ :

$$
\frac{\xi_{m, j}^{n+1}-\xi_{m, j}^{n}}{\Delta t^{n}}+\left(\bar{U}_{0}+\frac{1}{\lambda_{m}}\right) \frac{\xi_{m, j}^{n}-\xi_{m, j-1}^{n}}{\Delta x}-f v_{m, j}^{n}=F_{\xi, m, j}^{n}-B_{\xi, m, j}^{n}
$$

$$
\frac{v_{m, j}^{n+1}-v_{m, j}^{n}}{\Delta t^{n}}+\bar{U}_{0} \frac{v_{m, j}^{n}-v_{m, j-1}^{n}}{\Delta x}+f \frac{\xi_{m, j}^{n}+\eta_{m, j}^{n}}{2}=F_{v, m, j}^{n}-B_{v, m, j}^{n},
$$

$$
\frac{\eta_{m, j}^{n+1}-\eta_{m, j}^{n}}{\Delta t^{n}}+\left(\bar{U}_{0}-\frac{1}{\lambda_{m}}\right) \frac{\eta_{m, j}^{n}-\eta_{m, j-1}^{n}}{\Delta x}-f v_{m, j}^{n}=F_{\eta, m, j}^{n}-B_{\eta, m, j}^{n}
$$

Either $\xi_{m, 0}^{n+1}, v_{m, 0}^{n+1}$ and $\eta_{m, 0}^{n+1}$ are given by the boundary conditions defined as in [RTT05b] (transparent boundary conditions case), or they satisfy the periodicity conditions (4.2) below (periodical case). 
For every function $f(x, z, t), f_{m, j}^{n}$ represents $f_{m}\left(x_{j}, t_{n}\right)$ for $0 \leq j \leq J$, $0 \leq n \leq n_{\max }$, with

$$
\begin{array}{r}
0=x_{0}<x_{1}<\ldots<x_{j}<\ldots<x_{J}=L \\
0=t_{0}<t_{1}<\ldots<t_{n}<\ldots<t_{n_{\max }}=T \\
\Delta x=x_{j+1}-x_{j}=\frac{L}{J} \\
\Delta t^{n}=t_{n+1}-t_{n} .
\end{array}
$$

In the numerical experiments, we choose an homogeneous space discretization $(\Delta x=$ const $=L / J)$. For the sake of simplicity, we choose an explicit time-scheme, with a constant time-step $\Delta t$, which will be restricted by the well-known CFL condition to guarantee stability in the linear case:

$$
\Delta t \leq \frac{\Delta x}{\max _{1 \leq m \leq M}\left(\bar{U}_{0}, \bar{U}_{0}+\frac{1}{\lambda_{m}},\left|\bar{U}_{0}-\frac{1}{\lambda_{m}}\right|\right)}=\frac{\Delta x}{\bar{U}_{0}+\frac{1}{\lambda_{1}}} .
$$

Naturally, when the equations are nonlinear, the CFL condition is not enough to guarantee stability. Actually, the characteristic values depend on time since $\bar{U}_{0}$ has to be replaced by $u+\bar{U}_{0}$, but we assume that the initial data is such that $\left|u_{0}\right|<<\bar{U}_{0}$, which is physically relevant, [TT03]. We actually base our computations on those of the quoted article [TT03]: in this article the initial data is such that the ratio between the perturbation and the reference flow $\bar{U}_{0} \mathbf{e}_{\mathbf{x}}$ is less than $10 \%$, which is physically relevant. In the case of numerical simulations with periodic boundary conditions, we multiply the initial data of [TT03] by $\sin (\pi x / L)$ to make it periodic and avoid any boundary layer at $t=0$.

\section{Numerical Simulations}

We present hereafter two different sets of numerical results. In Section 4.1 we resolve the nonlinear PEs in a domain $\Omega_{0}=(0, L) \times(-H, 0)$ with periodic boundary conditions in the horizontal $(x)$ direction, and $w=0$ at $z=-H, 0$. These numerical results will provide the boundary conditions needed for the computations of Section 4.2 below, and we evidence in Section 4.3 the transparent properties of the boundary conditions introduced, thanks to a comparison between the solutions computed in Section 4.1 and those of Section 4.2.

The computations are done as follows. We fix $M$ (the number of modes) and compute $\left(u_{m}^{0}, v_{m}^{0}, \psi_{m}^{0}\right)_{0<m \leq M}$ from the given data $u^{0}, v^{0}, \psi^{0}$ thanks to (3.1)-(3.2).

Then, for every mode $m \leq M$, we consider the modal equations (2.20) 
and their discretization (3.5)-(3.6), and supplement them with the appropriate boundary conditions, either (4.3) for the periodical case or (4.7)-(4.8) for the case of transparent boundary conditions. We recall here that for every $m,\left(\xi_{m}, \eta_{m}\right)=\left(u_{m}-\psi_{m} / \lambda_{m}, u_{m}+\psi_{m} / \lambda_{m}\right)$ will be the numerical unknowns to be computed. , so that $\left(u_{m}, w_{m}, \psi_{m}, \phi_{m}\right)$ can be obtained with

$$
\begin{array}{r}
u_{m}(x, t)=\frac{\xi_{m}+\eta_{m}}{2}(x, t), \\
w_{m}(x, t)=-\frac{u_{m_{x}}}{N \lambda_{m}}(x, t), \\
\psi_{m}(x, t)=\frac{N\left(\eta_{m}-\xi_{m}\right)}{2}(x, t), \\
\phi_{m}(x, t)=-\frac{\psi_{m}}{N \lambda_{m}}(x, t) .
\end{array}
$$

As a consequence, we will only consider the quantities $\left(\xi_{m}, v_{m}, \eta_{m}\right)$ in the sequel, the other physical quantities beeing easily computed thanks to $(4.1)$.

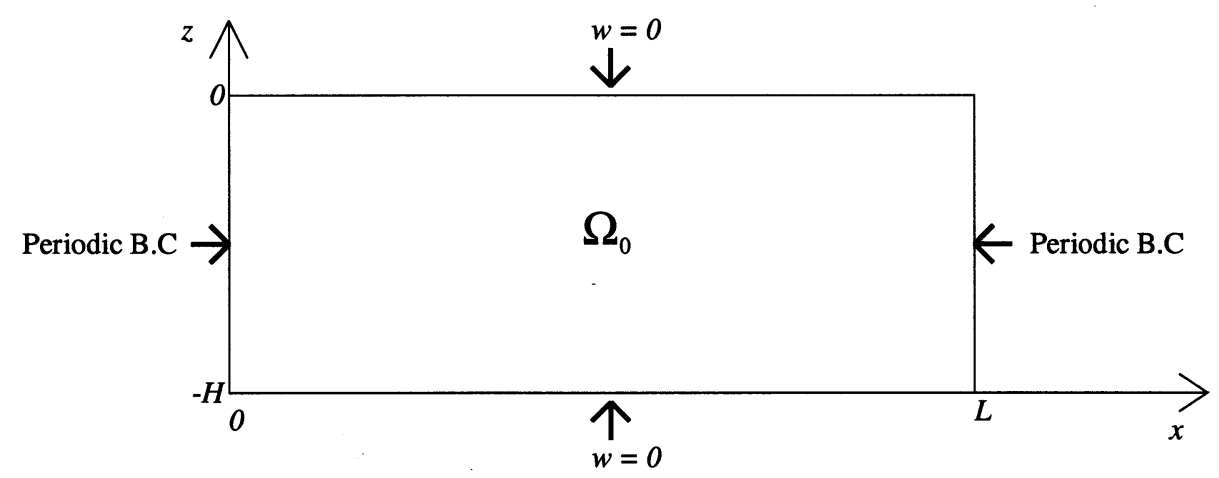

FiguRE 3. Domain $\Omega_{0}$

4.1. Periodic boundary conditions for the large domain $\Omega_{0}$. In the periodical case, we consider the following modal boundary conditions:

$$
\begin{aligned}
& \xi_{m}(0, t)=\xi_{m}(L, t), \\
& v_{m}(0, t)=v_{m}(L, t), \\
& \eta_{m}(0, t)=\eta_{m}(L, t) .
\end{aligned}
$$

For each time step $\Delta t^{n}=\Delta t$ satisfying (3.11) we compute the unknown functions $\left(\xi_{m}^{n+1}, v_{m}^{n+1}, \eta_{m}^{n+1}\right)$ thanks to (3.5) and (3.6), with the 
numerical boundary conditions:

$$
\begin{aligned}
\xi_{m, 0}^{n+1} & =\xi_{m, J}^{n+1}, \\
v_{m, 0}^{n+1} & =v_{m, J}^{n+1}, \\
\eta_{m, 0}^{n+1} & =\eta_{m, J}^{n+1} .
\end{aligned}
$$

The following figures plot $u, v$ and $\psi$ in the domain $\Omega_{0}$ at two different times. Figures 4,5 and 6 represent the initial data $(t=0)$ for these three quantities, whereas Figures 7,8 and 9 represent $u, v$ and $\psi$ at $t=t_{1}>0$.
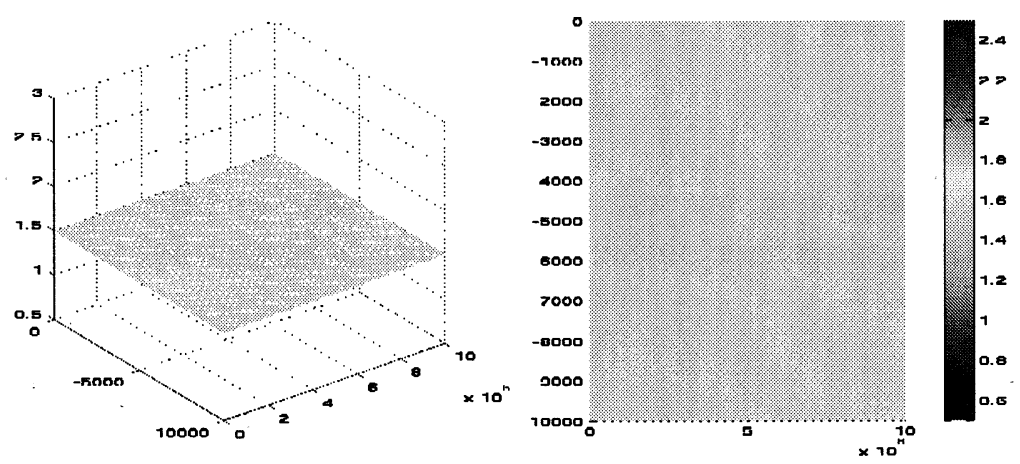

Figure 4. Periodic Boundary Condition. Initial data $u_{0}$. 
NUMERICAL SIMULATIONS OF THE INVISCID PRIMITIVE EQUATIONS 15
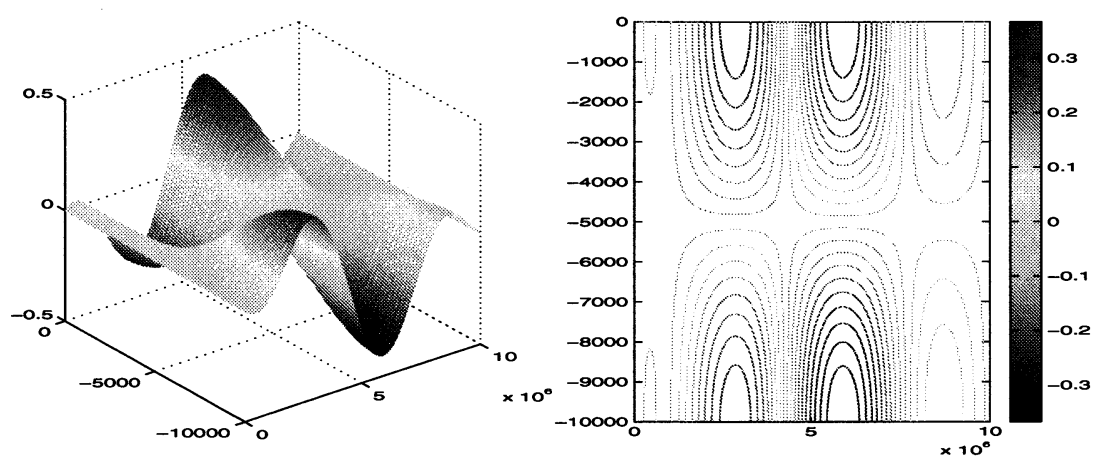

Figure 5. Periodic Boundary Condition. Initial data $v_{0}$.
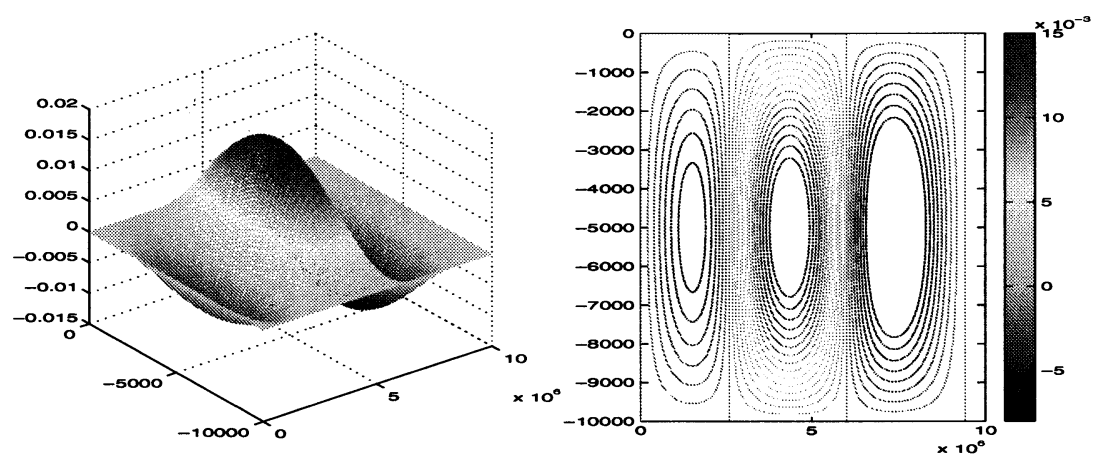

Figure 6. Periodic Boundary Condition. Initial data $\psi_{0}$.
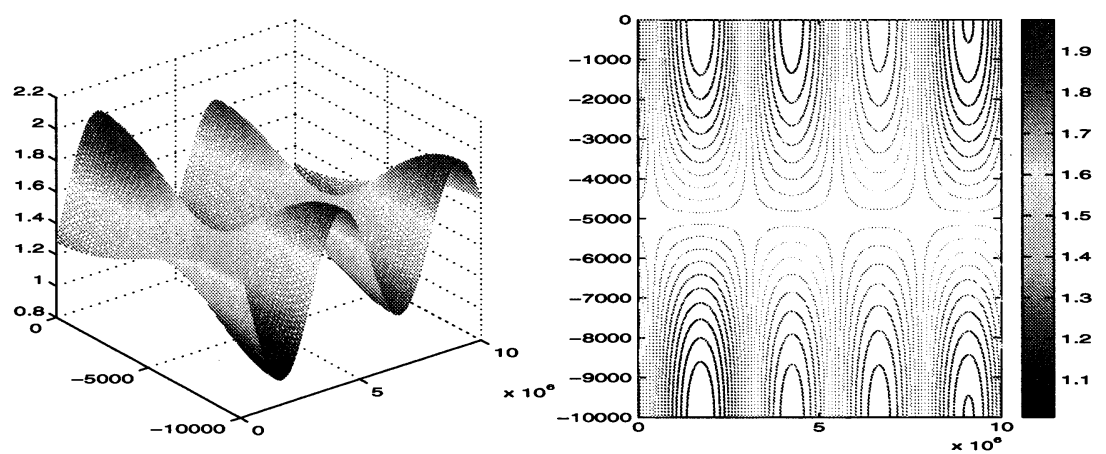

Figure 7. Periodic Boundary Condition. Values of $u$ at $t=t_{1}$. 

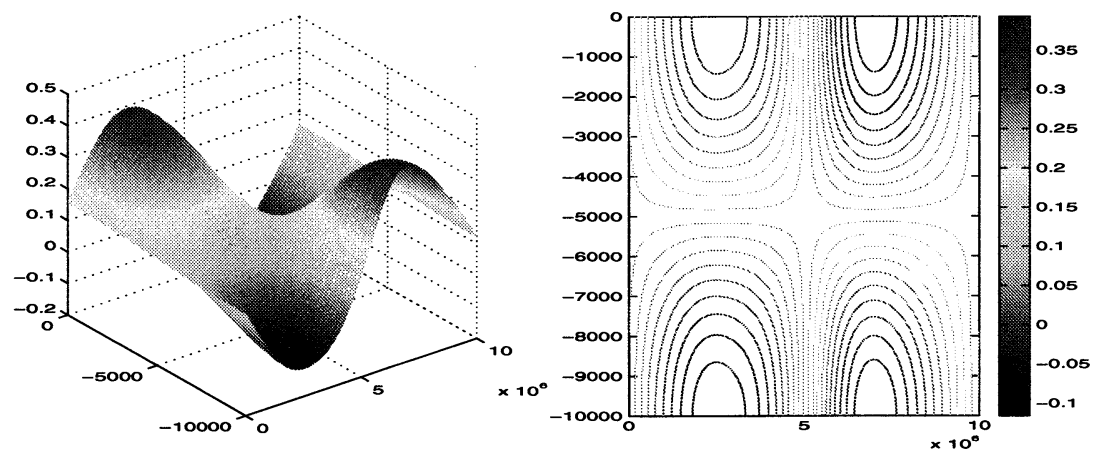

FiguRE 8. Periodic Boundary Condition. Values of $v$ at $t=t_{1}$.

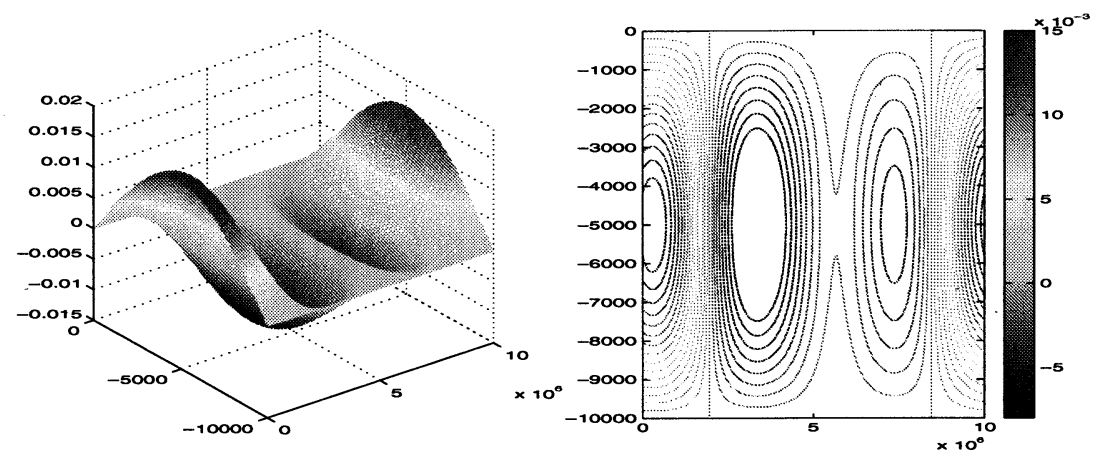

Figure 9. Periodic Boundary Condition. Values of $\psi$ at $t=t_{1}$.

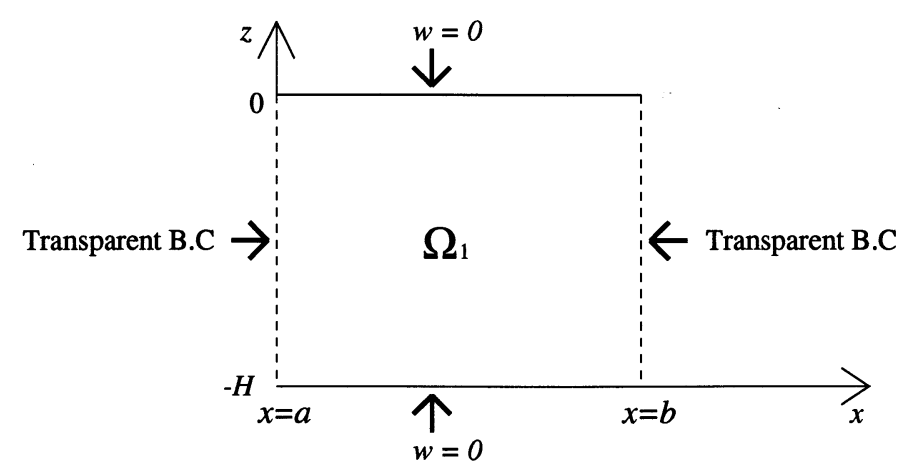

FiguRE 10. Subdomain $\Omega_{1}$

4.2. Transparent boundary conditions for the subdomain $\Omega_{1} \subset$ $\Omega_{0}$. We now intend to simulate the PEs in the subdomain $\Omega_{1}=(a, b) \times$ 
$(-H, 0)$ and the boundary conditions are the nonlinear version of those introduced in [RTT05b]. In the numerical simulations below we will consider a domain $\Omega_{1}=(a, b) \times(-H, 0)$ such that $0<a<b<L$, so that $\Omega_{1}$ is actually imbedded in $\Omega_{0}=(0, L) \times(-H, 0)$. The space discretization is now changed to $x_{j}=a+j(b-a) / J, 0 \leq j \leq J$.

At the boundaries $x=a$ and $x=b$, we will consider the nonhomogeneous form of the transparent boundary conditions of [RTT05b]. We use the computations of Section 3.1 above to provide the right-handside of the boundary conditions (4.5) and (4.6) below, and afterwards use them for comparison in the subdomain $\Omega_{1}$. These boundary conditions, expressed in a general way, are given in [RTT05b]. They consist in an infinite set of integral boundary conditions. For example:

$$
\int_{-H}^{0} v(a, z, t) \mathcal{U}_{m}(z) d z=\int_{-L_{3}}^{0} \tilde{v}(a, z, t) \mathcal{U}_{m}(z) d z, \quad \forall m \leq M
$$

where $\tilde{U}=(\tilde{u}, \tilde{v}, \tilde{w}, \tilde{\psi}, \tilde{\phi})$ are some known functions, computed in the domain $\Omega_{0}$ with some periodic boundary conditions (see Section 3.1 above).

Hence, for every subcritical mode $\left(m \leq m_{c}\right)$ and every time $t>0$, we have:

$$
\begin{aligned}
& \xi_{m}(a, t)=\tilde{\xi}_{m}(a, t), \\
& v_{m}(a, t)=\tilde{v}_{m}(a, t), \\
& \eta_{m}(b, t)=\tilde{\eta}_{m}(b, t),
\end{aligned}
$$

where $\tilde{\xi}_{m}$ and $\tilde{\eta}_{m}$ are defined as usual.

For the supercritical modes, we set for every $t>0$ :

$$
\begin{aligned}
& \xi_{m}(a, t)=\tilde{\xi}_{m}(a, t), \\
& v_{m}(a, t)=\tilde{v}_{m}(a, t), \\
& \eta_{m}(a, t)=\tilde{\eta}_{m}(a, t) .
\end{aligned}
$$

To implement these boundary conditions, we discretize equations (2.20) with the finite differences method, taking into account the sign of $\bar{U}_{0}-$ $1 / \lambda_{m}$ for the discretization of the first $x$-derivative of $\eta_{m}$ in equation (2.20c) (see equations (3.5) and (3.6) of Section 3 above).

For each time step $\Delta t^{n}=\Delta t$ satisfying (3.11) we compute the unknown functions $\left(\xi_{m}^{n+1}, v_{m}^{n+1}, \eta_{m}^{n+1}\right)$ thanks to (3.5) and (3.6), with the 
numerical boundary conditions:

$$
\begin{aligned}
\xi_{m, 0}^{n+1} & =\tilde{\xi}_{m}\left(a, t_{n+1}\right), \\
v_{m, 0}^{n+1} & =\tilde{v}_{m}\left(a, t_{n+1}\right), \\
\eta_{m, J}^{n+1} & =\tilde{\eta}_{m}\left(b, t_{n+1}\right),
\end{aligned}
$$

if $m$ is subcritical $\left(m \leq m_{c}\right)$. If $m$ is supercritical $\left(m>m_{c}\right)$, we set

$$
\begin{aligned}
& \xi_{m, 0}^{n+1}=\tilde{\xi}_{m}\left(a, t_{n+1}\right), \\
& v_{m, 0}^{n+1}=\tilde{v}_{m}\left(a, t_{n+1}\right), \\
& \eta_{m, 0}^{n+1}=\tilde{\eta}_{m}\left(a, t_{n+1}\right) .
\end{aligned}
$$

The following figures plot $u, v$ and $\psi$ in the domain $\Omega_{1}$ at two different times. Figures 11, 12 and 13 represent the initial data $(t=0)$ for these three quantities, whereas Figures 14,15 and 16 represent $u, v$ and $\psi$ at $t=t_{1}>0$.

Here, one can see that Figures 14, 15 and 16 respectively match with Figures 7,8 and 9 in the domain $\Omega_{1}$.

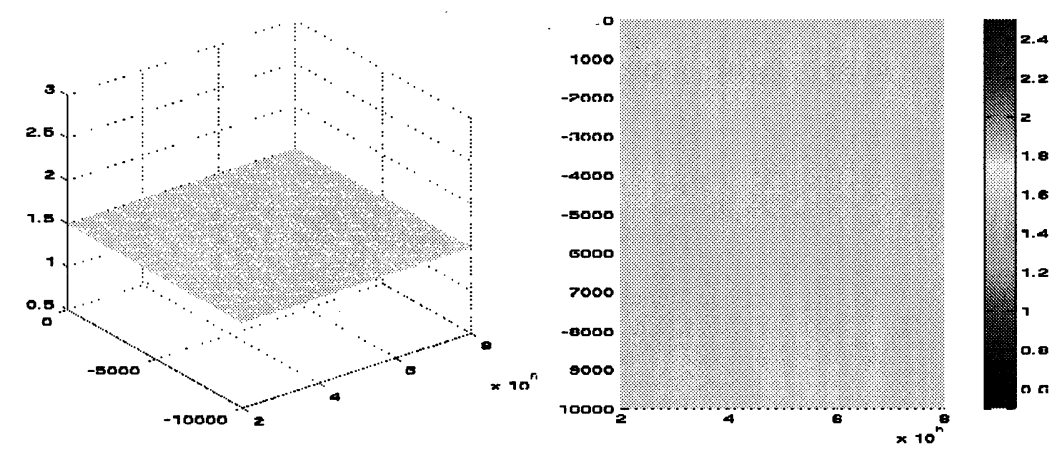



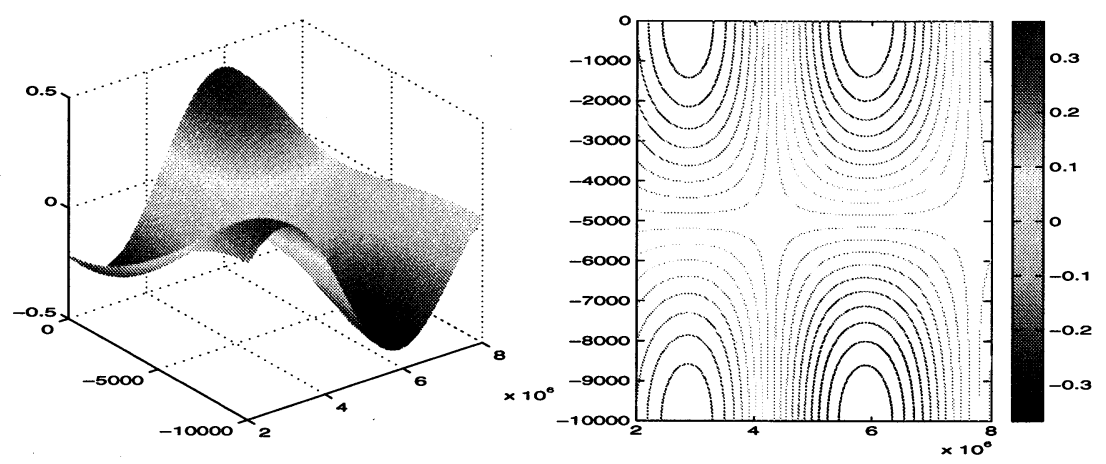

FIgURE 12. Transparent Boundary Condition. Initial data $v_{0}$.
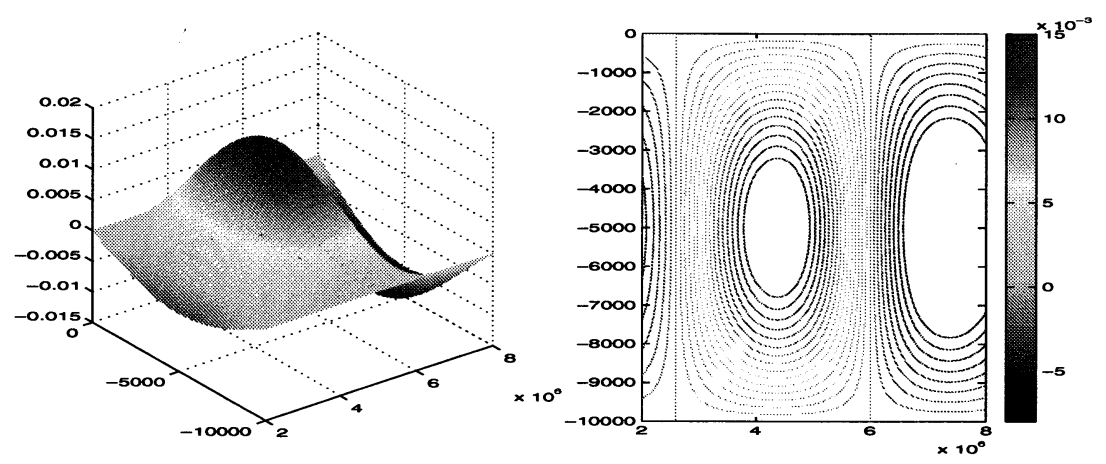

FIGURE 13. Transparent Boundary Condition. Initial data $\psi_{0}$.
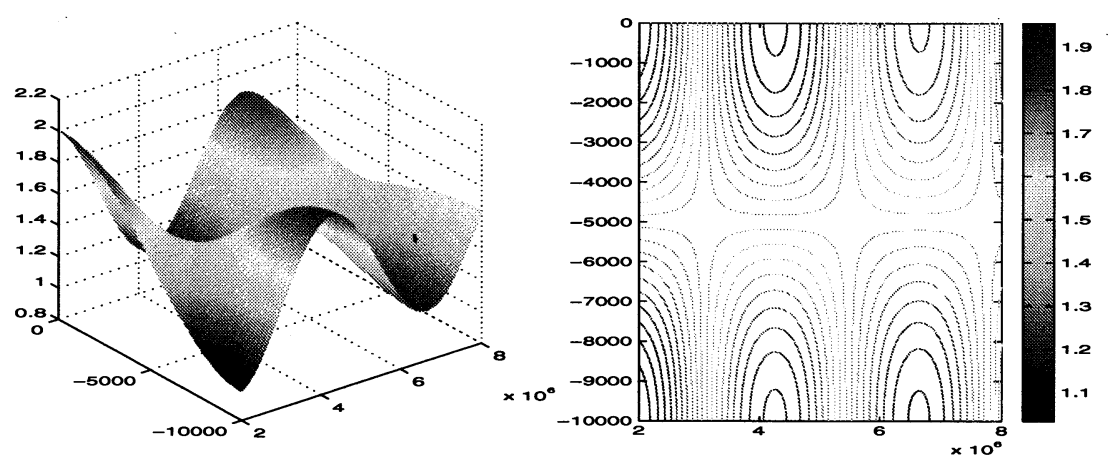

FiguRe 14. Transparent Boundary Condition. Values of $u$ at $t=t_{1}$. 

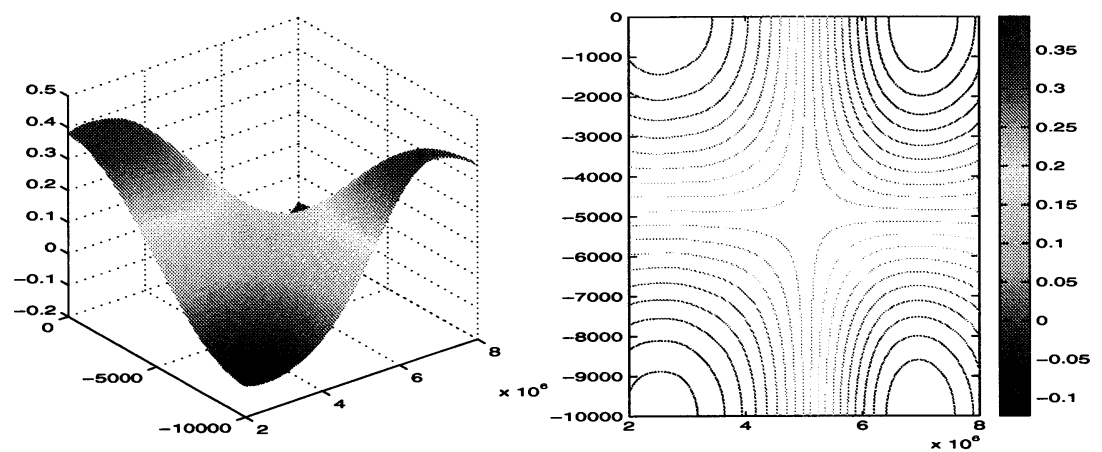

FIGURE 15. Transparent Boundary Condition. Values of $v$ at $t=t_{1}$.
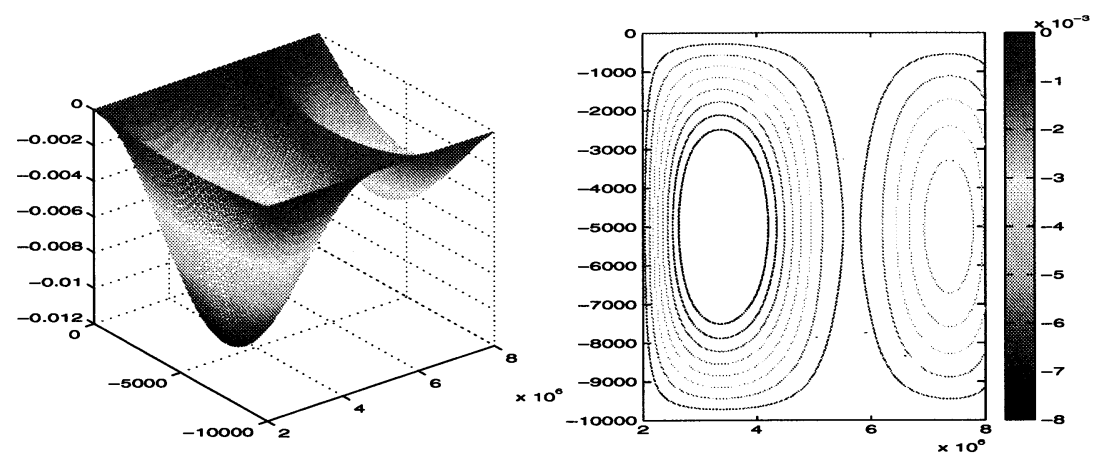

FiguRE 16. Transparent Boundary Condition. Values of $\psi$ at $t=t_{1}$.

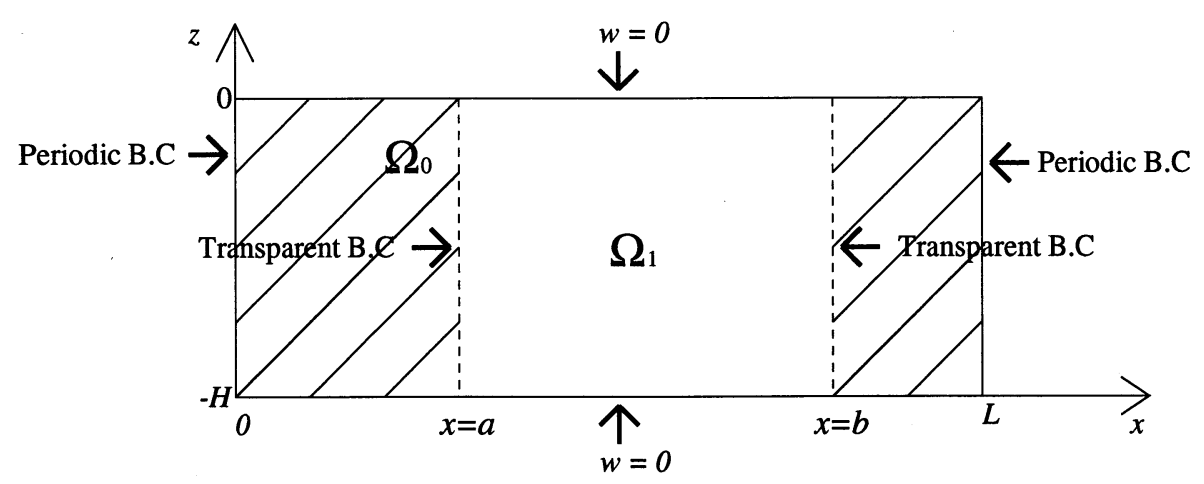

FiguRE 17. Subdomains $\Omega_{0}$ and $\Omega_{1}$

4.3. Comparisons. In order to confirm what can be observed, we finally choose an interior point $\left(x_{0}, z_{0}\right)=\left(5.8 \times 10^{6},-4.0 \times 10^{3}\right) \in \Omega_{1}$, and plot in Figure 18 the values of $(u, v, \psi)\left(x_{0}, z_{0}, t\right)$ computed in $\Omega_{1}$ with transparent boundary conditions, compared to $(u, v, \psi)\left(x_{0}, z_{0}, t\right)$ 
computed in $\Omega_{0}$ with periodic boundary conditions. The results are similar if one considers another choice of $\left(x_{0}, z_{0}\right)$; this shows the transparency property of the boundary conditions (4.5)-(4.6).
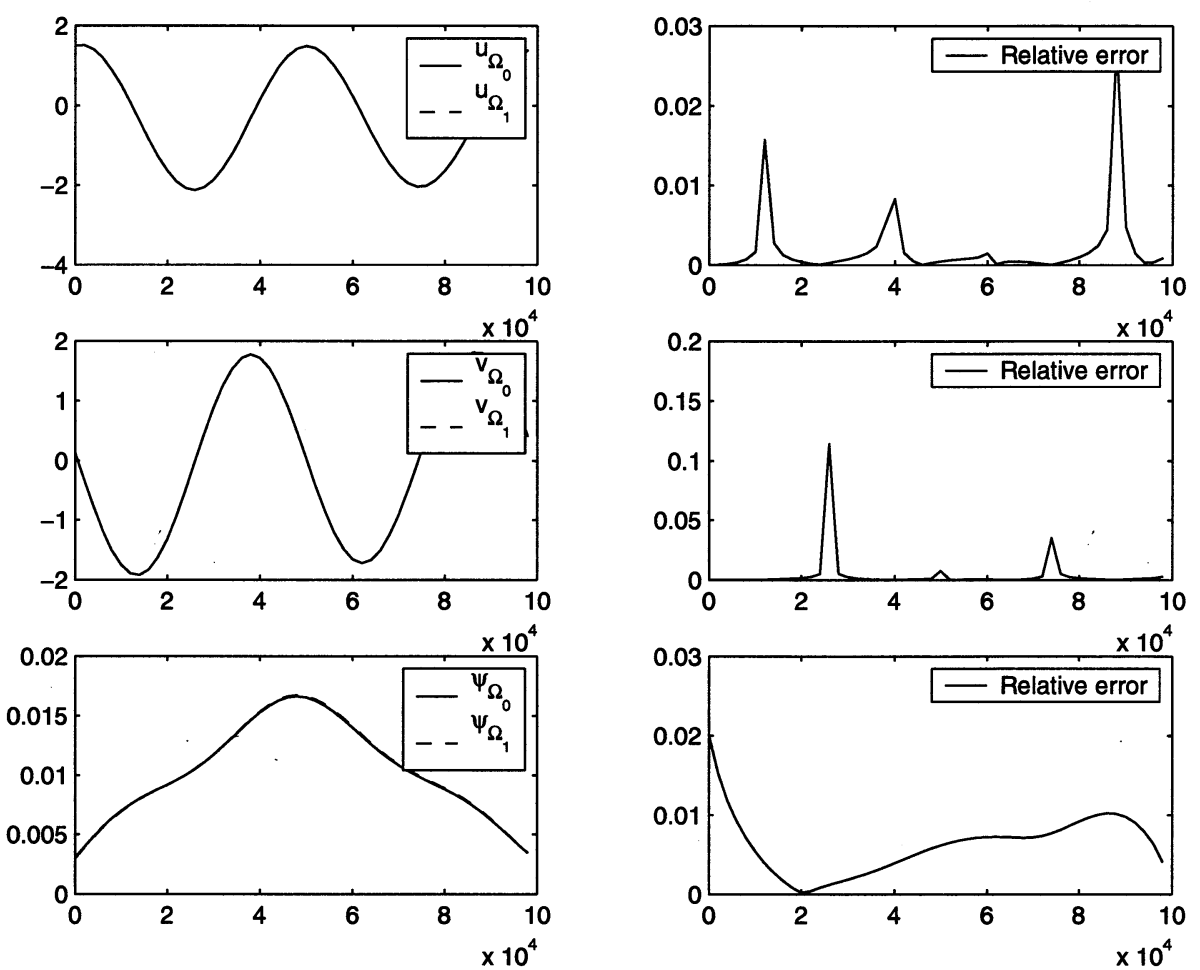

FIgURE 18. Two different computations of $\psi\left(x_{0}, z_{0}, t\right)$

(left). Relative error (right).

On the left part, we plot the different quantities $(u, v, \psi)\left(x_{0}, z_{0}, t\right)$ computed with the two types of boundary conditions. On the right part, we plot the corresponding relative errors $\left|f_{\Omega_{0}}-f_{\Omega_{1}}\right| /\left|f_{\Omega_{0}}\right|$ where $f$ is successively $u, v$ and $\psi$. The reader might think that the relative error reaches some local high values, but this is actually due to the fact that the quantity $u_{\Omega_{0}}$ (or $v_{\Omega_{0}}, \psi_{\Omega_{0}}$ ) vanishes; these local maximum are not meaningful.

\section{Conclusion}

In this article, the primitive equations of the ocean are considered. After recalling the study of the linearized version of these equations, we present some numerical results on the nonlinear system of equations. The boundary conditions that are implemented are those of the linear problem, which is physically relevant, at least for some time. 
We achieve here two goals: firstly we started here the extension of the theoretical results of [RTT05b] to the nonlinear primitive equations. Secondly the proposed boundary conditions are well-suited for the problem of numerical simulations in a limited domain, as shown by the very good matching of the two different numerical solutions.

\section{REFERENCES}

[BM97] C. Bernardi and Y. Maday. Spectral methods. In Handbook of numerical analysis, Vol. V, Handb. Numer. Anal., V, pages 209-485. NorthHolland, Amsterdam, 1997.

[GH01] D. Gottlieb and J. S. Hesthaven. Spectral methods for hyperbolic problems. J. Comput. Appl. Math., 128(1-2):83-131, 2001. Numerical analysis 2000, Vol. VII, Partial differential equations.

[OS78] J. Oliger and A. Sundström. Theoretical and practical aspects of some initial boundary value problems in fluid dynamics. SIAM J. Appl. Math., 35(3):419-446, 1978.

[PR05] M. Petcu and A. Rousseau. On the $\delta$-primitive and Boussinesq type equations. Advances in Differential Equations, to appear, 2005.

[RTT05a] A. Rousseau, R. Temam, and J. Tribbia. Boundary conditions for an ocean related system with a small parameter. In Nonlinear PDEs and Related Analysis, volume 371, pages 231-263. Gui-Qiang Chen, George Gasper and Joseph J. Jerome Eds, Contemporary Mathematics, AMS, Providence, 2005.

[RTT05b] A. Rousseau, R. Temam, and J. Tribbia. Boundary conditions for the 2D linearized PEs of the ocean in the absence of viscosity. Discrete and Continuous Dynamical Systems, to appear, 2005.

[TT03] R. Temam and J. Tribbia. Open boundary conditions for the primitive and Boussinesq equations. J. Atmospheric Sci., 60(21):2647-2660, 2003. 